\title{
Lack of increases in methylation at three CpG-rich genomic loci in non-mitotic adult tissues during aging
}

\author{
Michelle W Chu'1, Kimberly D Siegmund ${ }^{2}$, Carrie L Eckstam¹, \\ Jung Yeon Kim ${ }^{1,6}$, Allen S Yang ${ }^{3}$, Gary C Kanel ${ }^{1}$, Simon Tavaré ${ }^{4,5}$ and \\ Darryl Shibata*1
}

\begin{abstract}
Address: ${ }^{1}$ Department of Pathology, University of Southern California Keck School of Medicine, Los Angeles, CA 90033, USA, ${ }^{2}$ Department of Preventive Medicine, University of Southern California Keck School of Medicine, Los Angeles, CA 90033, USA, ${ }^{3}$ Department of Medicine, University of Southern California Keck School of Medicine, Los Angeles, CA 90033, USA, ${ }^{4}$ Department of Biological Sciences, University of Southern California, Los Angeles, CA 90089, USA, ${ }^{5}$ Department of Oncology, University of Cambridge, Cambridge, UK and ${ }^{6}$ Department of Pathology, Inje University Sanggye-Paik Hospital, Sanggye 7 dong 761-7, Nowon-gu, Seoul, Korea

Email: Michelle W Chu - waichu@usc.edu; Kimberly D Siegmund - kims@usc.edu; Carrie L Eckstam - carrieeckstam@gmail.com; Jung Yeon Kim - pck2973@unitel.co.kr; Allen S Yang - yang_a@ccnt.hsc.usc.edu; Gary C Kanel - gckanel@aol.com;

Simon Tavaré - stavare@usc.edu; Darryl Shibata* - dshibata@usc.edu

* Corresponding author
\end{abstract}

Published: 31 July 2007

BMC Medical Genetics 2007, 8:50 doi:10.1 186/147I-2350-8-50
Received: 16 July 2007

Accepted: 31 July 2007

This article is available from: http://www.biomedcentral.com/I47/-2350/8/50

(c) 2007 Chu et al; licensee BioMed Central Ltd.

This is an Open Access article distributed under the terms of the Creative Commons Attribution License (http://creativecommons.org/licenses/by/2.0), which permits unrestricted use, distribution, and reproduction in any medium, provided the original work is properly cited.

\begin{abstract}
Background: Cell division occurs during normal human development and aging. Despite the likely importance of cell division to human pathology, it has been difficult to infer somatic cell mitotic ages (total numbers of divisions since the zygote) because direct counting of lifetime numbers of divisions is currently impractical. Here we attempt to infer relative mitotic ages with a molecular clock hypothesis. Somatic genomes may record their mitotic ages because greater numbers of replication errors should accumulate after greater numbers of divisions. Mitotic ages will vary between cell types if they divide at different times and rates.

Methods: Age-related increases in DNA methylation at specific CpG sites (termed "epigenetic molecular clocks") have been previously observed in mitotic human epithelium like the intestines and endometrium. These CPG rich sequences or "tags" start unmethylated and potentially changes in methylation during development and aging represent replication errors. To help distinguish between mitotic versus time-associated changes, DNA methylation tag patterns at 8-20 CpGs within three different genes, two on autosomes and one on the Xchromosome were measured by bisulfite sequencing from heart, brain, kidney and liver of autopsies from 21 individuals of different ages.

Results: Levels of DNA methylation were significantly greater in adult compared to fetal or newborn tissues for two of the three examined tags. Consistent with the relative absence of cell division in these adult tissues, there were no significant increases in tag methylation after infancy.

Conclusion: Many somatic methylation changes at certain $C_{P G}$ rich regions or tags appear to represent replication errors because this methylation increases with chronological age in mitotic epithelium but not in nonmitotic organs. Tag methylation accumulates differently in different tissues, consistent with their expected genealogies and mitotic ages. Although further studies are necessary, these results suggest numbers of divisions and ancestry are at least partially recorded by epigenetic replication errors within somatic cell genomes.
\end{abstract}




\section{Background}

Human "age" can be characterized by chronological age (time since the zygote) and mitotic age (total number of divisions since the zygote). Somatic cells within an individual have similar chronological ages but their mitotic ages may differ because different cell types divide at different rates and times. Cell division occurs during normal human development and aging, and many diseases are associated with excessive numbers of divisions. Despite the likely importance of cell division to human pathology, it has been difficult to measure mitotic ages because human lifetimes are long and direct observations or experimental manipulations are impractical.

Here we attempt to infer relative somatic cell mitotic ages with a molecular clock approach [1]. Divisions should be surreptitiously recorded within a genome because the greater the number of divisions, the greater the number of replication errors. Molecular clock approaches are commonly employed to study species evolution and infer ancestral trees. All life is thought to be related to a universal ancestor that existed billions of years ago [2]. Sequences differ between individuals and species because genomes cannot be duplicated exactly, and replication errors accumulate. Similarly, all cells within an individual are related, share an ancestral genome, and eventually trace their ancestry back to a universal common ancestor called the zygote (Figure 1). Somatic replication errors are likely to accumulate as genomes are copied during development and aging. Sequence comparisons could be used to infer the mitotic ages of human cells, but sequences mutate too infrequently during a lifetime to effectively function as somatic molecular clocks. For example, point mutations are rare ( $<1$ per 100,000 bases) even in cancer cell lines [3]. With this error frequency, one would have to sequence millions of bases to identify a few somatic replication errors in normal tissues. Microsatellite loci mutate at higher frequencies and various investigators have proposed or provided data illustrating their potential to reconstruct somatic cell ancestry $[4,5]$.

It may be possible to replace the $5^{\prime}$ to $3^{\prime}$ order of bases with the 5' to $3^{\prime}$ order of CpG DNA methylation because genome replication also involves the duplication of epigenetic patterns. Methylation also exhibits somatic inheritance [6], but is a binary (methylated or unmethylated) code. Unlike sequences, methylation measurably changes with age in certain mitotic human tissues at specific CpG sites [7], suggesting epigenetic replication error rates may be greater than genetic replication error rates.

DNA methylation patterns may be directly compared between different aged individuals because methylation is removed early in development (before implantation) at most CpG islands $[8,9]$. This early active and passive

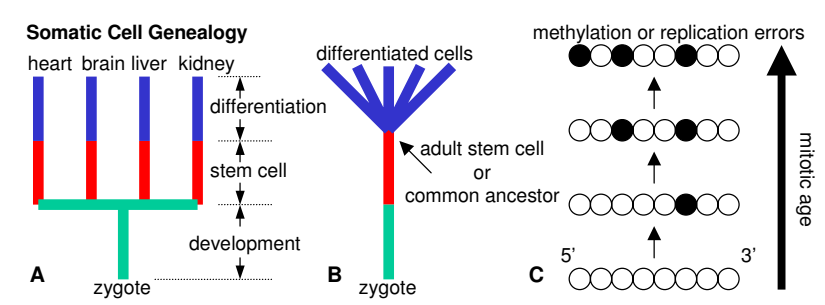

Figure I

A human somatic cell tree. A: Every cell has a genealogy that starts with the zygote and ends with its present day phenotype. The genealogy of a differentiated cell can be divided into three phenotypic phases - development from the zygote, a stem cell phase, and differentiation. B: Stem cells are common ancestors in a somatic cell tree. The zygote is the ultimate common ancestor, and adult stem cells are more recent common ancestors of differentiated cells. C: Replication errors record cell division or genome duplication. Illustrated are a 5' to 3' series of eight CpG sites that are initially unmethylated (open circles). Random replication errors may accumulate during cell division such that some CPG sites become methylated (filled circles). The greater the numbers of divisions since the zygote (mitotic age), the greater the number of errors or methylation (molecular clock hypothesis).

demethylation essentially synchronizes epigenomes between individuals and potentially an age-related increase in subsequent methylation at certain $\mathrm{CpG}$ rich regions ("epigenetic molecular clocks") may represent an accumulation of epigenetic replication errors. Bisulfite sequencing studies of mitotically active epithelium like human endometrium, colon, and small intestines reveal age-related methylation consistent with a "clock-like" accumulation of stochastic replication errors [10-13].

Replication errors may represent the major source of agerelated methylation because genomes are regularly duplicated in mitotic tissues. In addition, cell division appears to be required for in vitro de novo methylation [14]. Potentially the mitotic history of any cell can be reconstructed from its methylation patterns if such patterns are not "created" but instead represent a lifelong accumulation of replication errors. Cells with greater mitotic ages would generally have more errors or methylation relative to cells with fewer divisions. However, some methylation may occur independent of division, which would confound a somatic cell clock analysis. To further test whether methylation at "epigenetic molecular clocks" occurs independent of division, brain, heart, kidney, and liver tissues from individuals of different ages were examined. Cell division is minimal in adult brain and heart cells $[15,16]$, and age-related methylation should be absent in these tissues if the majority of tag methylation represents replica- 
tion errors. Adult liver and kidney cells normally divide infrequently but can divide in response to damage $[17,18]$.

\section{Methods \\ Specimens}

Specimens were obtained from 21 autopsies performed at the Los Angeles County-University of Southern California Medical Center (Table 1). The ethical use of the human tissues for research was approved by our Institutional Review Board. Intervals between death and tissue collection were generally less than three days. Heart specimens from 18 individuals were obtained from grossly normal right or left ventricle. Brain specimens from 18 individuals were obtained from grossly normal frontal cortex (primarily gray matter) or cerebellum. Liver specimens from 12 individuals were grossly normal except for the cirrhotic livers from Patients M and Q. Kidney specimens from 18 individuals were obtained from the cortex and appeared grossly normal.

DNA was purified from approximately 25 to $50 \mathrm{mg}$ of tissue using a column method (Dneasy, Qiagen, Valencia, CA). Approximately $500 \mathrm{ng}$ of DNA was bisulfite treated for 12 hours at $50 \mathrm{C}$ using an agarose bead method [11]. Bead sizes were about 25 to $30 \mathrm{ul}$.

\section{Methylation analysis}

Approximately $10 \%$ of the agarose bead containing bisulfite treated DNA was amplified for each PCR (42 cycles) of three different $\mathrm{CpG}$ rich loci or tags (Figure 2 and Table 2) that previously exhibited age-related methyl- ation in epithelial tissue $[11,19]$. The first tag is in the $3^{\prime}$ transcribed but untranslated region of the CSX gene (also called NKX2-5). This 51 bp tag contains 8 CpG sites and exhibits age related methylation in epithelial tissues such as the colon, small intestines, and endometrium [11-13]. The second tag is a 151 bp region in exon 2 of SOX10 that contains $20 \mathrm{CpG}$ sites. The SOX10 tag appears to be a relatively fast somatic cell molecular clock because it becomes nearly fully methylated in hair by two years of age [19]. Finally, a 77 bp region in exon 1 of BGN just 3' to the promoter that contains $9 \mathrm{CpG}$ sites and exhibits age related methylation in the colon [11] was examined in the heart and brain of males. The BGN tag is on the X-chromosome, and there is only a single allele per cell in males. PCR products were visualized on an agarose gel and cloned (TOPO TA Cloning kit, Invitrogen, Carlsbad, CA). Individual bacterial clones were sequenced to obtain seven or eight tags per locus per specimen. Clones with evidence of incomplete bisulfite conversion (C's at nonCpG sites) were discarded from the analysis.

Percent methylation is calculated by dividing numbers of methylated CpG sites by total numbers of examined CpG sites. Statistical analysis used linear regression to test for age-related changes in methylation, and a two-sided t-test for differences between specimen groups. Significance was set at $\mathrm{p}<0.05$.

\section{Results}

The specimens examined in this study are described in Table 1. Methylation was examined at three CpG rich regions or tags (Figure 2 and Table 2). These tags were ini-

Table I: Specimens

\begin{tabular}{|c|c|c|c|c|c|c|c|}
\hline Patient & Age & Sex & Cause of death & Brain & Heart & Kidney & Liver \\
\hline A & 20 wks & M & Stillborn & $x$ & $\mathrm{X}$ & $\mathrm{X}$ & $\mathrm{X}$ \\
\hline $\mathrm{B}$ & 22 wks & M & Hydrops fetalis (parvovirus) & $x$ & $\mathrm{X}$ & $\mathrm{X}$ & \\
\hline $\mathrm{C}$ & 32 wks & $\mathrm{F}$ & Congenital syphilis & $x$ & $\mathrm{X}$ & $\mathrm{X}$ & $\mathrm{X}$ \\
\hline $\mathrm{D}$ & 0 yrs & M & Stillborn, nuchal Cord & $\mathrm{X}$ & $\mathrm{X}$ & $\mathrm{X}$ & $\mathrm{X}$ \\
\hline $\mathrm{E}$ & 5 yrs & $M$ & Inborn error of metabolism & $\mathrm{X}$ & $x$ & $x$ & $x$ \\
\hline $\mathrm{F}$ & 25 yrs & $\mathrm{F}$ & Cerebral aneurysm & $x$ & $\mathrm{X}$ & $\mathrm{X}$ & \\
\hline G & 31 yrs & M & Testicular cancer, bleomycin toxicity & & & $\mathrm{x}$ & $\mathrm{X}$ \\
\hline $\mathrm{H}$ & 33 yrs & $M$ & Cholangiocarcinoma & $X$ & $\mathrm{x}$ & $\mathrm{X}^{*}$ & \\
\hline I & 36 yrs & $\mathrm{F}$ & Cardiomyopathy & & & & $x$ \\
\hline $\mathrm{J}$ & 39 yrs & $M$ & Alcoholic cirrhosis & $\mathrm{X}$ & $x$ & $\mathrm{x}$ & \\
\hline $\mathrm{K}$ & 41 yrs & $M$ & Blunt force trauma & $x$ & $x$ & $\mathrm{x}$ & \\
\hline $\mathrm{L}$ & 48 yrs & $M$ & AIDS & $x$ & $\mathrm{X}$ & $\mathrm{X}$ & $\mathrm{X}$ \\
\hline$M$ & 52 yrs & $M$ & Alcoholic cirrhosis & $x$ & $x$ & $\mathrm{X}^{*}$ & $\mathrm{X}^{* *}$ \\
\hline$N$ & 53 yrs & $M$ & Lung cancer & & & $\mathrm{X}$ & \\
\hline $\mathrm{O}$ & 54 yrs & $\mathrm{F}$ & Myocardial infarction & $x$ & $x$ & & $x$ \\
\hline $\mathrm{P}$ & 58 yrs & $\mathrm{F}$ & Breast cancer & $x$ & $x$ & & \\
\hline $\mathrm{Q}$ & 62 yrs & $M$ & Systemic amyloidosis/cirrhosis & $x$ & $\mathrm{X}$ & $X^{*}$ & $X^{* *}$ \\
\hline $\mathrm{R}$ & 70 yrs & M & Sepsis & $x$ & $\mathrm{X}$ & $X^{*}$ & $\mathrm{X}$ \\
\hline $\mathrm{S}$ & 72 yrs & $M$ & Systemic amyloidosis & $x$ & $\mathrm{X}$ & $\mathrm{X}$ & $\mathrm{X}$ \\
\hline $\mathrm{T}$ & 81 yrs & $M$ & Diabetes type II & $x$ & $\mathrm{x}$ & $X^{*}$ & $\mathrm{x}$ \\
\hline$\cup$ & 84 yrs & $\mathrm{F}$ & Gastric cancer & $x$ & $\mathrm{X}$ & $X^{*}$ & \\
\hline
\end{tabular}

\footnotetext{
* benign nephrosclerosis present

** cirrhosis present
} 


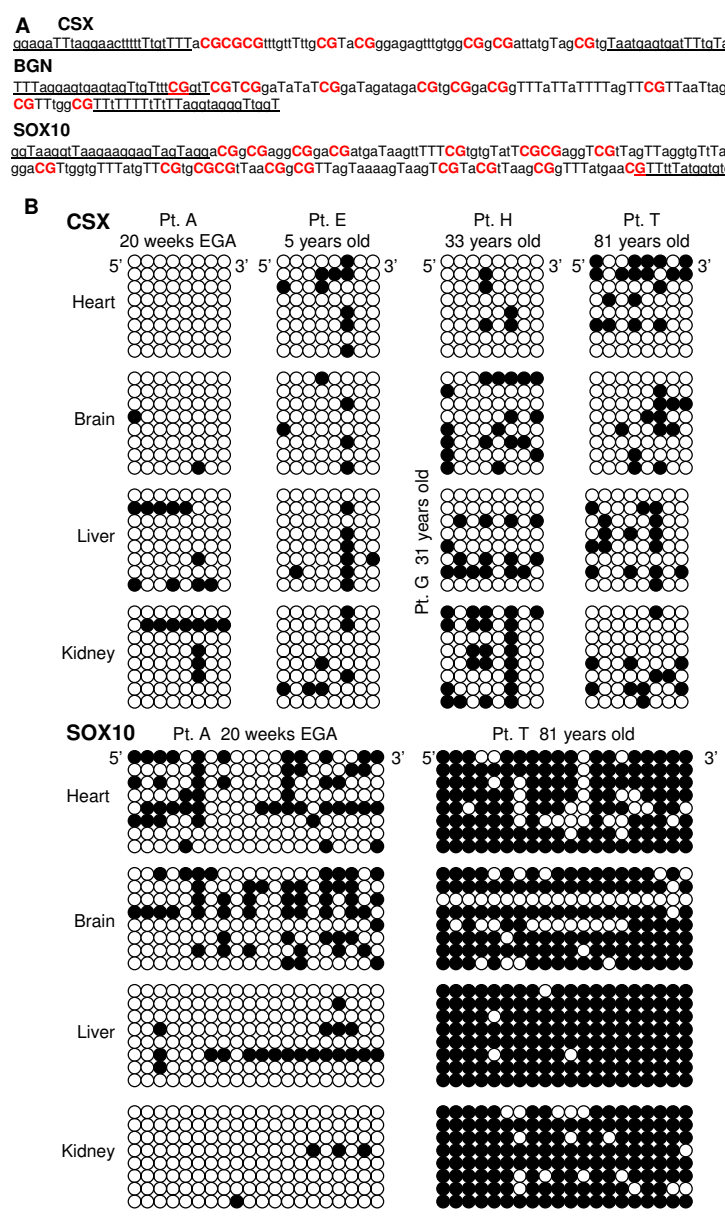

Figure 2

Epigenetic molecular clocks or tags. A: Sequences (after bisulfite conversion) of the CSX (8 CpG sites), BGN ( 9 CpG sites), and SOXI0 (20 CpG sites) tags. Primer sites are underlined, $C_{p G}$ sites are in bold, and a bisulfite converted "C" at a non-CpG site is indicated by "T". B: Examples of CSX and SOX 10 tags sampled from the heart, brain, liver or kidney of different aged individuals. The tags are arranged in a 5 ' to 3' horizontal order with methylated sites indicated by filled circles. There are eight alleles per tissue. Patient $\mathrm{H}$ did not have a liver specimen and the liver is instead from Patient $\mathrm{G}$, a 31 year old. tially chosen because they exhibit age-related methylation in epithelial tissues and their genes are not expressed at high levels in epithelium. However, some of the tags are expressed at higher levels in the tissues examined in this study (Table 2). CSX is highly expressed in the heart [20] and BGN has higher expression in the brain and heart. Expression may prevent methylation regardless of cell division because promoter methylation is associated with gene silencing [21]. Of note, only the BGN tag is located close to its promoter in exon 1 .

\section{Mitotic age and stochastic replication errors}

Mitotic age cannot be directly inferred from tag methylation because of the random or stochastic nature of replication errors. For example, methylation replication error rates for the CSX tag have been estimated at about $10^{-5}$ to $10^{-4}$ per CpG site per division [11]. Therefore, many cells will lack tag methylation even though they have divided many times. Cells with tag methylation may have either fewer, the same, or greater numbers of divisions than cells with less tag methylation.

The inability to link methylation levels directly with numbers of cell divisions is illustrated by simulation for CSX and SOX10 tags (Figure 3). For a given mitotic age, individual tag methylation patterns and values are scattered. However, average values of eight tags are less scattered. Therefore, the experimental studies are based on average measurements of seven or eight tags per specimen. Consistent with stochastic errors, tissue methylation patterns were diverse (Figure 2).

\section{Heart}

The heart continues to grow in size after birth, at rates comparable to body weight [22]. CSX and SOX10 tag methylation was significantly lower in fetal, infant and children ( $\leq 5$ years old) hearts and greater in adult $(>21$ years old) hearts (Figure 4 and Table 3). Age-related changes in CSX or SOX10 methylation were not observed in adult hearts. BGN heart tag methylation also did not exhibit age-related changes and was generally low $(<5 \%)$, with no significant differences between adult and young ( $\leq 5$ years old) hearts. There were no significant differences in tag methylation between the right and left heart.

Table 2: Expression of tag loci*

\begin{tabular}{ccccccccc}
\hline Gene & Chromosome & Location & Heart & Brain & Liver & Kidney & Colon & Uterus \\
\hline CSX & $5 q 34$ & last intron & $356^{* *}$ & 0 & 4 & 0 & 4 & 0 \\
SOX10 & $22 q 13.1$ & exon 2 & 0 & 0 & 0 & 0 & 0 & 4 \\
BGN & Xq28 & exon I & 401 & 246 & 85 & 653 & 183 & 740 \\
\hline
\end{tabular}

* data from website [29], search date 3/5/2007

** transcripts per million 


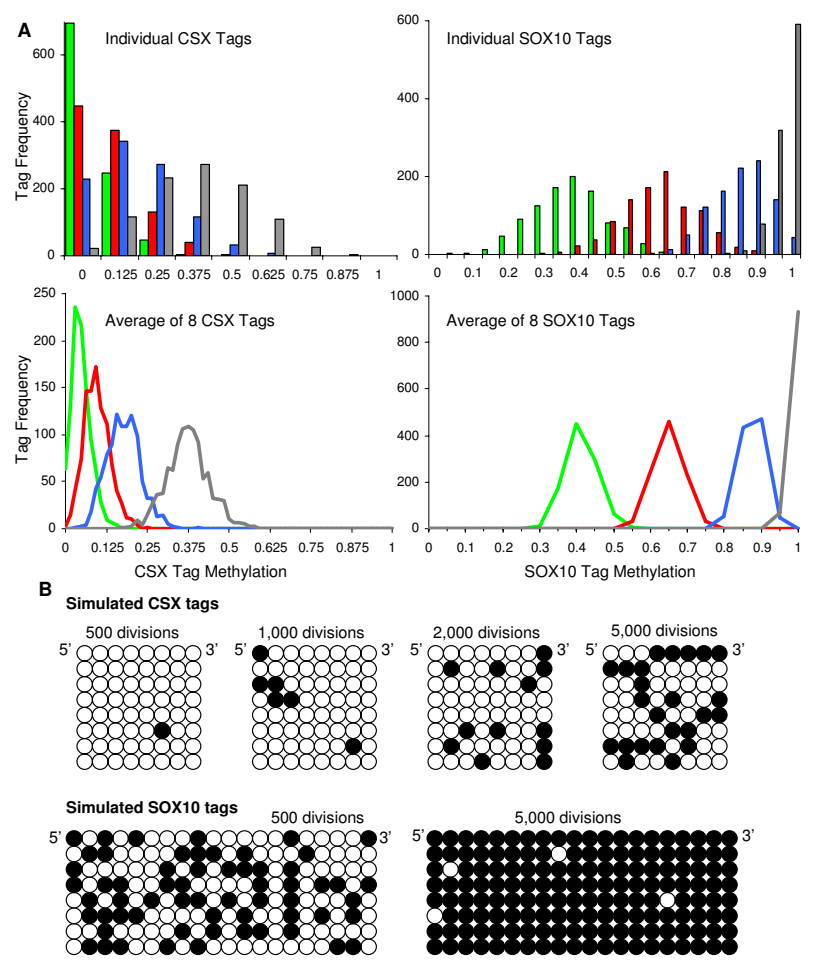

Figure 3

Simulations of random methylation errors at the CSX and SOX 10 tags. For CSX tags, the simulated error rates are $\mathrm{I} \times 10^{-4}$ per $\mathrm{C}_{\mathrm{pG}}$ site per division for methylation and $2 \times 10^{-5}$ for demethylation. SOX10 tags appear to become methylated faster in human tissues, and the simulated error rate for methylation is greater $\left(1 \times 10^{-3}\right)$ with the same demethylation error rate as CSX. Errors are assumed to be independent between $C_{P G}$ sites, and each tag is an independent genome (no shared ancestry). These simulations are presented to illustrate the general stochastic nature of mutation, and are not an attempt to model the experimental data. A: Frequencies of individual tag methylation values and averages of 8 tags after 500 (green), I,000 (red), 2,000 (blue), or 5,000 (gray) simulated divisions. As expected, values are more scattered for individual versus average tag values. B: Examples of simulated individual CSX or SOX 10 tags after different numbers of divisions.

\section{Brain}

Brain growth and development occurs early in development and is essentially complete by two years of age [15]. Statistically significant age-related changes in CSX, SOX10, or BGN tag methylation were not observed (Figure 5) after infancy (> 2 years of age). Significantly lower levels of CSX and SOX10 tag methylation were found in neonatal brains (Figure 5B and Table 3). CSX and SOX10 tag methylation levels were significantly greater in the cerebellum compared to the cerebral cortex (Figure 5C and

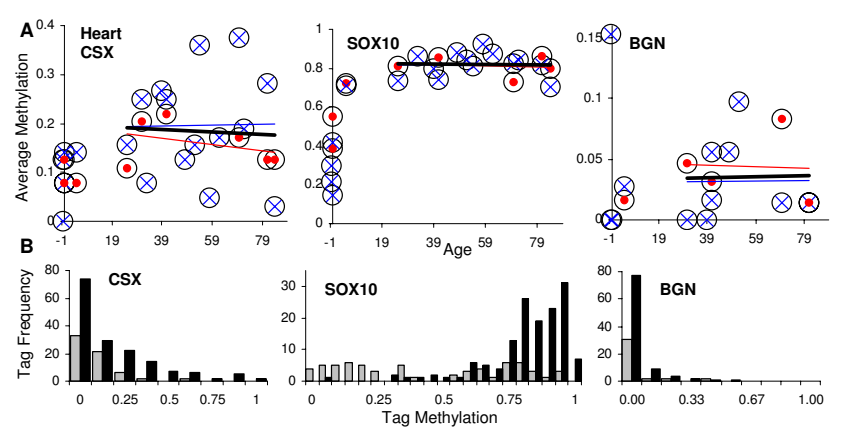

Figure 4

Heart tag methylation. A: Tag methylation with chronological aging. Circles are averages of eight tags with red solid dots from the right heart, and blue X's from the left heart. Trend lines (black, all values; red, right heart; blue, left heart) were not significantly different from a level line (linear regression, $p$ values $>0.05)$. B: Histograms of individual tags comparing individuals before heart growth is largely finished ( $\leq 5$ years of age, gray bars, $N=64,63$, and 37 alleles for CSX, SOXI0, and BGN tags) and adults (black bars, $N=16 \mathrm{I}$, I43, and 94 alleles for CSX, SOX I0, and BGN tags). CSX or SOXI0 tag methylation values were significantly higher in adults.

Table 3). However, there was no significant difference in BGN tag methylation between the cerebellum and cortex, or between neonatal and adult brains, but overall tag methylation levels were low $(<10 \%)$.

\section{Liver and kidney}

CSX and SOX10 kidney or liver tag methylation levels were significantly higher in adults relative to individuals less than 21 years of age (Figure 6 and Table 4). There was an age-related increase in tag methylation with adult kidneys or livers, but these trends were not statistically different from a level line. BGN tags were not examined in the kidney or liver.

Cirrhosis was present in two livers, one of which had the highest SOX10 tag methylation and high CSX tag methylation (Pt M). The other cirrhotic liver (Pt Q) had high CSX tag methylation but not remarkably high SOX10 methylation. The liver with the highest CSX tag methylation was from a patient with AIDS (Pt L), with no obvious liver pathology. The two kidneys with high CSX tag methylation had benign nephrosclerosis, which was not observed in kidneys from individuals of similar age with lower CSX methylation. Average CSX and SOX10 tag methylation was higher in kidneys with benign nephrosclerosis, but the average age of individuals with benign nephrosclerosis was also higher, consistent with the observation that nephrosclerosis is more common in the elderly [23]. 
Table 3: Heart and brain tag methylation

\begin{tabular}{|c|c|c|c|c|c|c|c|c|c|}
\hline \multirow[t]{2}{*}{ Tissue } & \multicolumn{3}{|c|}{ CSX methylation } & \multicolumn{3}{|c|}{ SOXI0 methylation } & \multicolumn{3}{|c|}{ BGN methylation } \\
\hline & $\leq 5$ yrs old & $>5$ yrs old & $p^{*}$ & $\leq 5$ yrs old & $>5$ yrs old & $p$ & $\leq 5$ yrs old & $>5$ yrs old & $p$ \\
\hline Heart & $9.6 \%(64)^{* *}$ & $18.3 \%(|6|)$ & $<0.001$ & $43.4 \%(63)$ & $81.7 \%(143)$ & $<0.001$ & $4.2 \%(37)$ & $3.6 \%(94)$ & 0.77 \\
\hline Right heart & & $15.9 \%(48)$ & 0.36 & & $81.1 \%(40)$ & 0.79 & & $4.4 \%(31)$ & 0.56 \\
\hline Left heart & & $19.4 \%(113)$ & & & $81.9 \%(103)$ & & & $3.2 \%(63)$ & \\
\hline & $<$ & & & $<$ & & & $<$ & & \\
\hline & $\leq 2$ yrs old & $>2$ yrs old & & $\leq 2$ yrs old & $>2$ yrs old & & $\leq 2$ yrs old & $>2$ yrs old & \\
\hline Brain & $3.8 \%(56)$ & $17.3 \%(160)$ & $<0.001$ & $44.4 \%(56)$ & $68.1 \%(160)$ & $<0.001$ & $5.6 \%(40)$ & $8.3 \%(104)$ & 0.34 \\
\hline Cortex & & $14.1 \%(1 \mid 2)$ & 0.005 & & $61.6 \%(112)$ & $<0.001$ & & $8.7 \%(72)$ & 0.71 \\
\hline Cerebellum & & $24.7 \%(48)$ & & & $83.1 \%(48)$ & & & $7.3 \%(32)$ & \\
\hline
\end{tabular}

* two sided t-test

** numbers of examined tags

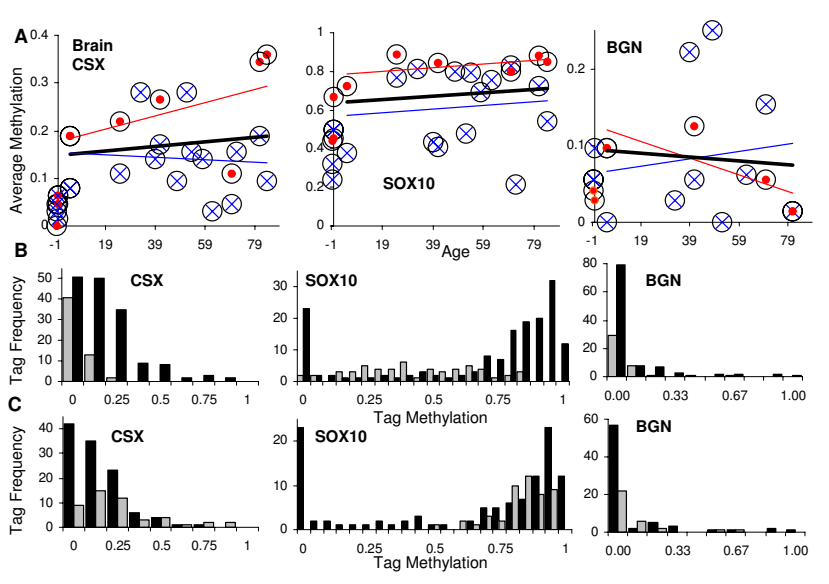

Figure 5

Brain tag methylation. A: Tag methylation with chronological aging. Circles are averages of eight tags with red dots from the cerebellum and blue X's from the cerebral cortex. Trend lines (black, all values; red, cerebellum; blue, cortex) were not significant different from a level line (linear regression, $\mathrm{p}$ values $>0.05$ ). B: Histograms of individual tags comparing individuals before brain development is largely finished ( $<2$ years of age, gray bars, $N=72,72$, and 56 alleles for CSX, SOXI0, and BGN tags) and older individuals $>2$ years of age (black bars, $\mathrm{N}=144,144$, and 88 alleles for CSX, SOX I0, and BGN tags). CSX or SOXI0 tag methylation values were significantly higher in older individuals. C: Histograms of individual tags comparing methylation between the cerebellum (gray bars, $\mathrm{N}=40,40$, and 24 alleles for CSX, SOX 10 , and BGN tags) and the cortex (black bars, $\mathrm{N}=104$, 104, and 64 alleles for CSX, SOX 10, and BGN tags) from older individuals ( $>2$ years of age). CSX and SOX 10 tags were significantly more methylated in the cerebellum. Note that there is a high frequency of completely unmethylated SOX 10 tags in the older cortex, which may reflect the presence of neural cells that express SOXIO and therefore preclude methylation (see Discussion).

\section{Tag changes in multiple tissues}

Trend lines for average CSX or SOX10 tag methylation with age for heart, brain, liver and kidney are illustrated in Figure 7A. For comparison with prior studies $[11-13,19]$, average CSX and SOX10 tag methylation with age are also illustrated for colon, small intestine, endometrium and hair in Figure 7B. In general, tag methylation is low around birth and higher in adults. Statistically significant age-related increases in CSX tag methylation were observed in colon, small intestines, and endometrium. SOX10 tags become methylated faster than CSX tags and age-related changes are not observed because they are nearly fully methylated in adult tissues, especially in mitotic epithelium.

\section{Discussion}

Although much is known about human development and aging, the exact fates of somatic cells are somewhat mysterious because it is difficult to observe cells throughout a lifetime. Potentially somatic cell mitotic ages may be inferred by counting numbers of replication errors using a molecular clock approach [10]. Here we provide further evidence that somatic genomes likely record divisions as they are duplicated and physically passed from generation to generation. Certain methylation patterns at specific CPG rich sequences or tags appear to represent somatic replication errors and therefore surreptitiously record mitotic ages.

Prior studies of mitotic adult tissues (intestines and endometrium) demonstrated age-related tag methylation consistent with methylation representing replication errors [11-13]. Cell division is initially required for normal heart and brain development but is infrequent in adulthood $[15,16]$. Consistent with tag methylation representing replication errors, adult brain and heart tags were methylated but failed to demonstrate age-related increases. The adult tag methylation likely represents rep- 

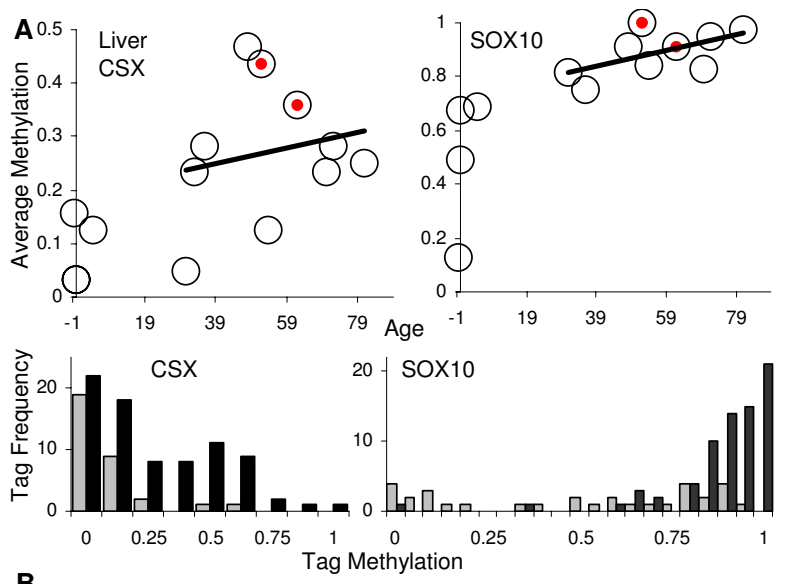

B
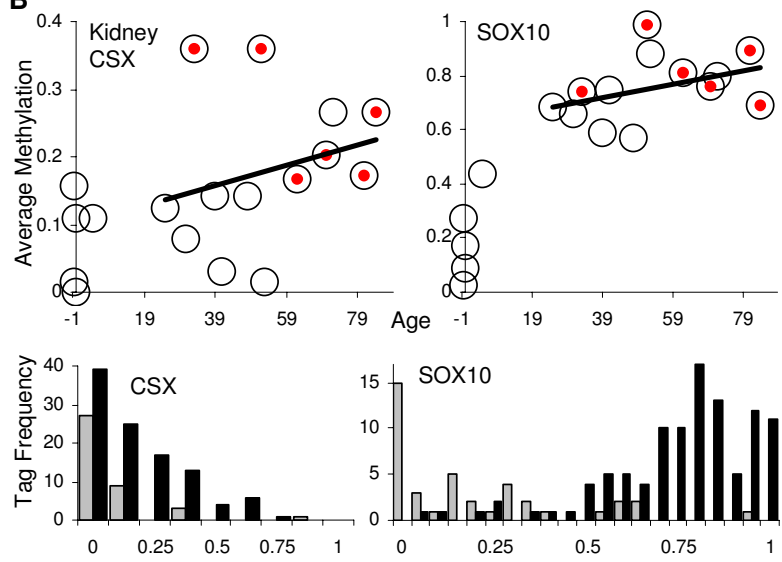

Figure 6

Liver and kidney methylation. A: Tag methylation with chronological aging in the liver. The trend line indicates an age-related increase in methylation, but the data were not significantly different from a level line $(p=0.38$ for CSX, $p=$ 0.19 for SOX 10, linear regression). Red solid dots indicate the two samples with cirrhosis. Tag methylation was significantly greater in adults (black bars, $\mathrm{N}=80$ and 72 alleles for CSX and SOX 10 tags) compared to younger individuals $\leq 5$ years of age (gray bars, $N=32$ alleles for CSX and SOX 10 tags). B: Tag methylation with chronological aging in the kidney. The trend line indicates an age-related increase in methylation, but the data were not significantly different from a level line ( $p=0.59$ for CSX, $p=0.097$ for SOX 10). Red solid dots indicate samples with benign nephrosclerosis. Tag methylation was significantly greater in adults (black bars, $N=105$ and 104 alleles for CSX and SOXI0 tags) compared to younger individuals $\leq 5$ years of age (gray bars, $N=40$ alleles for CSX and SOXIO tags). lication errors acquired much earlier in life during development because methylation was lower in fetal and infant tissues. Therefore, tag methylation patterns appear to be stable for decades in the absence of division.

Cell division occurs rarely in normal adult liver, perhaps once a year [17]. Consistent with low division rates, tag methylation increased with chronological age, but this trend was not significantly different from a level line. Relatively higher tag methylation levels were observed in livers with cirrhosis, consistent with observations that cirrhosis is associated with hepatocyte proliferation [24]. Division in normal kidney is thought to occur but is relatively rare [18]. Consistent with low mitotic activity, tag methylation apparently increased with age but this trend was not statistically significant. Relatively higher tag methylation was observed in kidneys with benign nephrosclerosis, a relatively common finding at autopsy that increases with age [23].

\section{Comparisons between different human tissues}

A molecular clock approach can compare genomes in cells of widely different phenotype. For example, sequences potentially infer ancestry across all three kingdoms of life [2]. Similarly, a somatic cell clock analysis may allow relative comparisons between cell types if their replication error rates are similar. CSX and SOX10 tag methylation with chronological age for multiple tissues are illustrated in Figure 7. Tag methylation was lower early in life for all tissues, consistent with a start with unmethylated tags and a common origin for their genomes. Methylation or errors increased early in life, consistent with the hyperplasia needed for growth and differentiation. Division largely ceases after birth for the brain or heart, and their mitotic ages and numbers of replication errors appear to remain fixed for a lifetime. In contrast to the heart, brain, liver and kidney, there were statistically significant age-related increases in methylation in the epithelial tissues [11-13]. Generally, endometrium from older women appears to have the highest average mitotic ages, with relatively lower mitotic ages in the heart and brain.

Different tags may have different replication error rates and attain different levels of methylation. Tags with higher error rates better document early development because the mitotic ages of neonatal and infant tissues are relatively low. For example, SOX10 tags become nearly fully methylated by infancy and therefore only minimal changes are possible even with further increases in mitotic age. By contrast, tags such as CSX with lower error rates exhibit fewer changes early in life but more readily document further mitotic age changes during aging. 
Table 4: Kidney and liver tag methylation

\begin{tabular}{|c|c|c|c|c|c|c|}
\hline \multirow[t]{2}{*}{ Tissue } & \multicolumn{3}{|c|}{ CSX methylation } & \multicolumn{3}{|c|}{ SOXI0 methylation } \\
\hline & $<21$ yrs old & $>21$ yrs old & $p^{*}$ & $<21$ yrs old & $>21$ yrs old & $P$ \\
\hline Kidney & $7.8 \%(40)^{* *}$ & $17.9 \%(105)$ & 0.003 & $19.9 \%(40)$ & $75.4 \%(104)$ & $<0.001$ \\
\hline Liver & $8.6 \%(32)$ & $27.2 \%(80)$ & $<0.001$ & $49.4 \%(32)$ & $88.7 \%(72)$ & $<0.001$ \\
\hline
\end{tabular}

* two sided t-test

** numbers of examined tags in parentheses.

\section{Human somatic cell genealogy}

Mitotic age contains additional information about somatic cell genealogy because only certain cell types divide during the differentiation from a zygote to present day cells. Somatic cell genealogy can be divided into three successive phenotypic phases - development from the zygote, a stem cell phase, and differentiation (Figure 1). Genealogy may be confined to certain patterns because numbers of divisions during development and differentiation may be restricted to characteristic numbers and times. We have observed methylation patterns consistent with three basic human genealogies (Figure 8).

The first pattern, observed in non-mitotic adult tissues such as the brain and heart, is a static genealogy. Mitotic

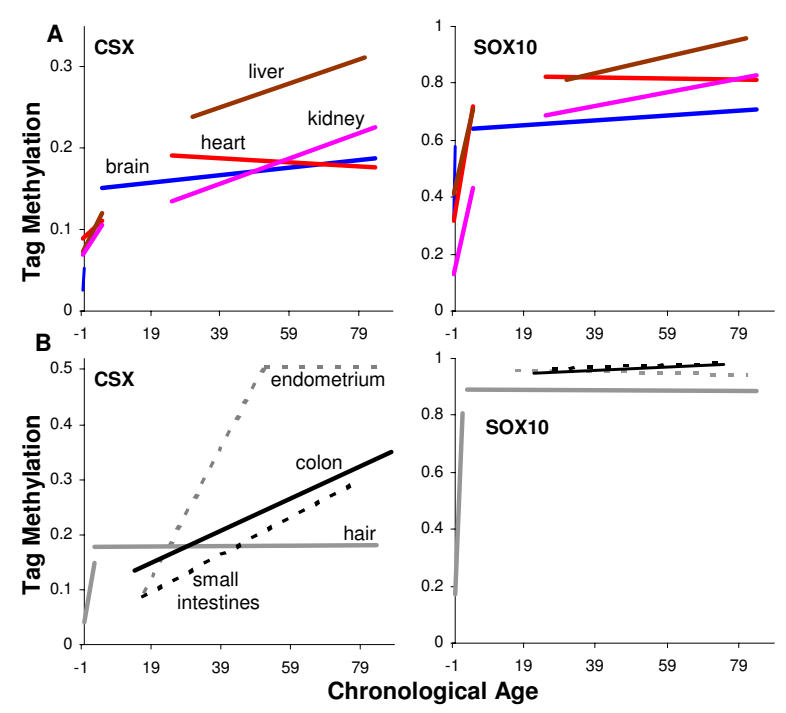

Figure 7

Average tag methylation with chronological age for multiple human tissues. A: Trend lines for brain (blue), heart (red), liver (brown), and kidney (purple). Negative ages represent fetal tissues (birth equal zero years). B: Trend lines for colon (black, data from ref. I I and additional unpublished specimens), small intestines (black dotted, data from ref. I2), endometrium (gray dotted, data from ref. 13), and hair follicles (gray, data from ref. 19). SOXI0 tag values for intestines and endometrium are unpublished data from three different aged individuals (six glands and 48 tags from each organ). age remains constant during chronological aging, and replication errors found in adult cells primarily represent divisions during earlier organ developmental and differentiation. Liver and kidney also have this general pattern, but further stem or differentiated cell divisions may occur during adult life from normal cell turnover or in response to cell loss.

A second pattern, observed in mitotic epithelium such as the intestines and endometrium, is a continuous genealogy that expands as replication errors accumulate throughout life. Replication errors likely accumulate primarily in adult stem cells because stem cell lineages are long lived and numbers of divisions during development and differentiation are usually similar regardless of chronological age. For example, intestinal development is limited to the neonatal period and differentiated cells in the intestines divide only a few times and survive about a week [25]. Therefore, only the stem cell phase can have variable numbers of divisions with aging. The mitotic age of a differentiated intestinal cell is the mitotic age of its stem cells plus the few divisions required for differentiation. Stem cells in the colon, small intestines, and endometrium appear to divide throughout life because their differentiated cells contain greater numbers of replication errors or methylation with increasing chronological age.

A third pattern, observed in hair follicles, is a punctuated genealogy. There is a lack of a measurable age-related increase in average replication errors despite high hair follicle mitotic activity [19]. This pattern may occur if stem cells exhibit mitotic latency, either by dividing infrequently or through clonal succession [26]. Human hair grows for several years and then is lost when the differentiated cells in the mitotic compartment or bulb physically disappears. A new follicle and hair regrow after several months, initiated by stem cells that reside in the hair bulge [27]. Bulge hair stem cells are normally quiescent and divide primarily at the start of a new hair cycle. This stem cell quiescence (infrequent division) or clonal succession (latent stem cells successively divide) ensures that the average mitotic age of hair bulb cells does not measurably increase with chronological age because each new hair cycle starts from a "young" stem cell. Mitotic age is reset when differentiated but mitotic bulb cells (and their 

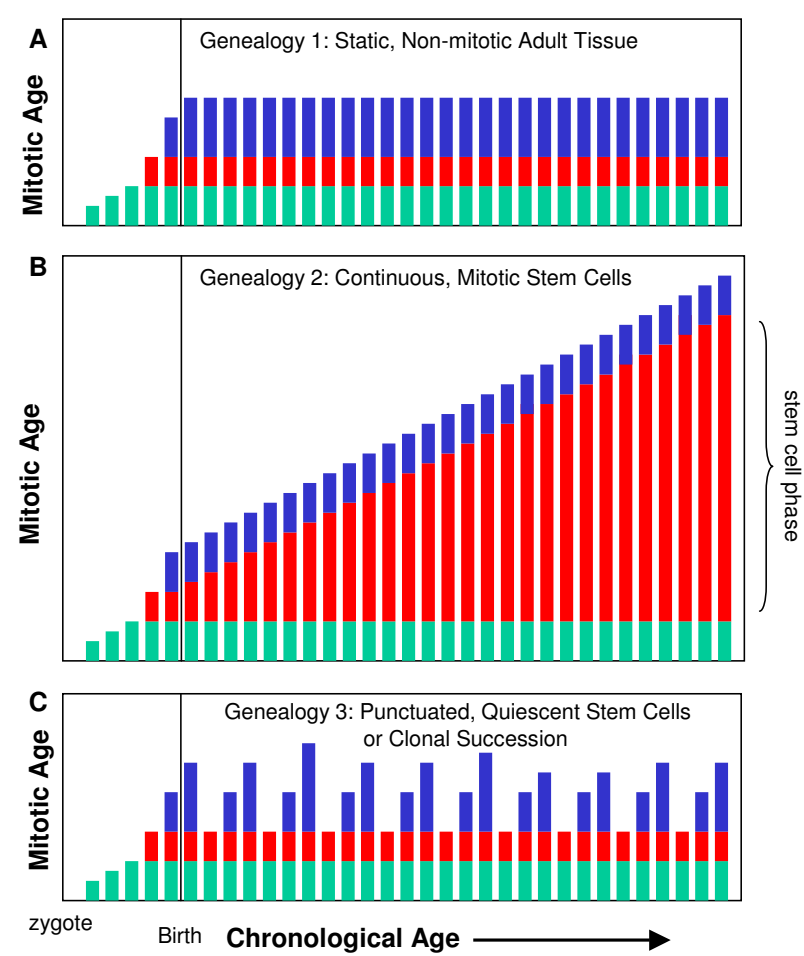

\section{Figure 8}

Types of observed human somatic cell genealogies.

A: Static genealogy observed in adult non-mitotic tissues (heart, brain, kidney, liver). Genealogical phases are indicated by green (development from the zygote to the tissue stem cell), red (stem cell phase), and blue (differentiation). Adult genealogies and mitotic ages are fixed with respect to chronological age and replication errors in present day cells accumulated much earlier in life. B: Continuous genealogy observed in mitotic epithelium. Only the stem cell phase can vary with chronological age because numbers of divisions are relatively constant during development and differentiation. The mitotic age of a differentiated cell is the mitotic age of its stem cell plus the few additional divisions required for differentiation. An age-related increase in replication errors and mitotic age depends on stem cell divisions. C: Punctuated genealogy observed in hair follicles. Average mitotic age and replication errors do not increase with age despite the high hair follicle mitotic activity because hairs and their follicles are cyclically lost and destroyed every few years. Bulge stem cells initiate each new hair cycle, and the mitotic age of a differentiated follicle cell is the age of the stem cell that initiates the new cycle plus the divisions in the follicle. New hairs would have essentially the same mitotic age regardless of chronological age because bulge stem cells are relatively quiescent and only divide a few times at the start of each new hair cycle [26], or if there is clonal succession [25] in which new hair cycles are initiated by previously latent stem cells. errors) are eliminated at the end of a hair cycle, and the next new hair has essentially the same genealogy regardless of chronological age. Physical configuration may influence genealogy, with a punctuated genealogy observed with episodic follicle destruction and reformation, and continuous genealogies observed with glands that persist throughout life.

\section{Epigenetic clocks and selection}

Selection can alter error frequencies by favoring or eliminating some types of errors. Methylation can potentially confer selection because promoter methylation is associated with loss of expression, and promoter methylation is another mechanism to silence tumor suppressor genes in cancer [21]. The loci studied (CSX, SOX10, BGN) were initially chosen because these genes were not highly expressed in epithelium and therefore any methylation would likely be neutral. However, some of these genes are expressed at higher levels in the heart and brain (Table 2), and evidence that expression prevents methylation was observed for the BGN tag, with relatively little methylation $(<10 \%)$ even in adult brain or heart. By contrast, CSX is highly expressed in the heart [20], but CSX tags appeared to record mitotic age with less methylation early in life and stable ( $18 \%)$ adult heart methylation. The CSX tag is in the 3' untranslated region of its gene, which may not affect or be affected by its transcription, whereas the BGN tag is just $3^{\prime}$ to the promoter in exon 1 . The SOX10 gene is expressed in only some neural tissues, mainly those of neural crest origin [28]. Fully unmethylated SOX10 tags were rarely found after birth in any tissue, except in the cerebral cortex (Figure 5), suggesting SOX10 expression in certain neural cells may preclude or prevent methylation in the region (exon 2) of our tag. Although the mechanism responsible for methylation at our tags is uncertain, methylation does appear to be reduced when the tag is located near an active promoter.

Replication errors are stochastic and the methylation of a single allele is relatively uninformative. Our analysis sampled a relatively small number of tags from only a few different loci, but illustrates the potential for "somatic cell clocks" to record mitotic ages. More information can be obtained with more tags from the same "clock" locus or from multiple "clock" loci. Methylation may not be independent between $\mathrm{CpG}$ sites and error rates may vary between CpG sites within a tag or between cell types. However, with most replication error mechanisms, average methylation should generally correlate with mitotic age. Another biological source for tag variation is the sampling of heterogeneous cell types. In this study, organ fragments containing uncertain mixtures of different cell types were analyzed. Ideally tags from small homogeneous clones of cells (as with our prior epithelial studies of 
single glands [11-13]) would be measured because each cell type may have a distinct genealogy.

\section{Conclusion}

The present study provides tentative evidence that somatic cell mitotic ages may be recorded within their genomes by methylation changes at certain $\mathrm{CpG}$ rich loci. The exact mechanisms responsible for methylation changes at our "epigenetic molecular clocks" are uncertain, but many changes appear to represent replication errors. Mitotic age in actively dividing adult tissues may largely depend on the behavior of their stem cells because numbers of divisions during development and differentiation are limited. There are a number of challenges to inferring genealogies from replication errors, but such an approach potentially allows for systematic studies of human development and aging without prior experimental interventions.

\section{Competing interests}

The author(s) declare that they have no competing interests.

\section{Authors' contributions}

MWC, CLE, and JYK performed most of the experiments and helped write the manuscript. ASY and GCK supplied specimens. KDS and ST helped with the statistical and quantitative analysis. DS analysed the data and wrote the manuscript.

\section{Acknowledgements}

This work was supported by a grant from the National Institutes of Health (CAII I 940). ST is a Royal Society-Wolfson Research Merit Award holder and is supported in part by a grant from Cancer Research UK.

\section{References}

I. Bromham L, Penny D: The modern molecular clock. Nat Rev Genet 2003, 4:216-224.

2. Woese C: The universal ancestor. Proc Natl Acad Sci USA 1998 , 95:6854-6859.

3. Wang TL, Rago C, Silliman N, Ptak J, Markowitz S, Willson JK, Parmigiani G, Kinzler KW, Vogelstein B, Velculescu VE: Prevalence of somatic alterations in the colorectal cancer cell genome. Proc Natl Acad Sci USA 2002, 99:3076-3080.

4. Salipante SJ, Horwitz MS: Phylogenetic fate mapping. Proc Natl Acad Sci USA 2006, 103:5448-5453.

5. Frumkin D, Wasserstrom A, Kaplan S, Feige U, Shapiro E: Genomic variability within an organism exposes its cell lineage tree. PLoS Comput Biol 2005, I(5):e50.

6. Holliday R: The inheritance of epigenetic defects. Science 1987, 238: $163-170$.

7. Issa JP: CpG-island methylation in aging and cancer. Curr Top Microbiol Immunol 2000, 249:101-118.

8. Bird A: DNA methylation patterns and epigenetic memory. Genes Dev 2002, 16:6-21.

9. Morgan HD, Santos F, Green K, Dean W, Reik W: Epigenetic reprogramming in mammals. Hum Mol Genet 2005, I 4:R47-58.

10. Shibata $D$, Tavaré $S$ : Counting divisions in a human somatic cell tree: how, what and why? Cell Cycle 2006, 5:6|0-6I4.

II. Yatabe $Y$, Tavaré $S$, Shibata D: Investigating stem cells in human colon by using methylation patterns. Proc Natl Acad Sci USA 200I, 98: 10839-10844.

12. Kim JY, Siegmund KD, Tavaré S, Shibata D: Age-related human small intestine methylation: evidence for stem cell niches. BMC Medicine 2005, 3:10.
13. Kim JY, Tavaré S, Shibata D: Counting human somatic cell replications: Methylation mirrors endometrial stem cell divisions. Proc Natl Acad Sci USA 2005, 102: 17739-17744.

14. Velicescu M, Weisenberger DJ, Gonzales FA, Tsai YC, Nguyen CT, Jones PA: Cell division is required for de novo methylation of CpG islands in bladder cancer cells. Cancer Res 2002, 62:2378-2384.

15. Dobbing J, Sands J: Quantitative growth and development of human brain. Arch Dis Child 1973, 48:757-767.

16. Anversa $P$, Nadal-Ginard B: Myocyte renewal and ventricular remodelling. Nature 2002, 415:240-243.

17. Sell S: Heterogeneity and plasticity of hepatocyte lineage cells. Hepatology 200I, 33:738-750.

18. Mene $P$, Polci R, Festuccia F: Mechanisms of repair after kidney injury. J Nephrol 2003, 16:186-195.

19. Kim JY, Tavaré S, Shibata D: Human hair genealogies and stem cell latency. BMC Biology 2006, 4:2

20. Komuro I, Izumo S: Csx: a murine homeobox-containing gene specifically expressed in the developing heart. Proc Natl Acad Sci USA 1993, 90:8I45-8I49.

21. Jones PA, Laird PW: Cancer epigenetics comes of age. Nat Genet 1999, 21:163-167.

22. Scholz DG, Kitzman DW, Hagen PT, Ilstrup DM, Edwards WD: Agerelated changes in normal human hearts during the first 10 decades of life. Part I (Growth): A quantitative anatomic study of $\mathbf{2 0 0}$ specimens from subjects from birth to 19 years old. Mayo Clin Proc 1988, 63:126-136.

23. Kasiske BL: Relationship between vascular disease and ageassociated changes in the human kidney. Kidney Int 1987, 31:1153-II59.

24. Greenbaum LE: Cell cycle regulation and hepatocarcinogenesis. Cancer Biol Ther 2004, 3: 1200-1207.

25. Potten CS, Loeffler M: Stem cells: attributes, cycles, spirals, pitfalls and uncertainties. Lessons for and from the crypt. Development 1990, II 0:1001-1020.

26. Kay HE: How many cell-generations? Lancet 1965, 15:418-419.

27. Fuchs E, Tumbar T, Guasch G: Socializing with the neighbors: stem cells and their niche. Cell 2004, I I6:769-778.

28. Mollaaghababa R, Pavan WJ: The importance of having your SOX on: role of SOX 10 in the development of neural crestderived melanocytes and glia. Oncogene 2003, 22:3024-3034.

29. UniGene EST ProfileViewer [http://www.ncbi.nlm.nih.gov/Uni Gene]

\section{Pre-publication history}

The pre-publication history for this paper can be accessed here:

http://www.biomedcentral.com/1471-2350/8/50/prepub

Publish with Bio Med Central and every scientist can read your work free of charge

"BioMed Central will be the most significant development for disseminating the results of biomedical research in our lifetime. "

Sir Paul Nurse, Cancer Research UK

Your research papers will be:

- available free of charge to the entire biomedical community

- peer reviewed and published immediately upon acceptance

- cited in PubMed and archived on PubMed Central

- yours - you keep the copyright 